\title{
ANÁLISIS CRÍTICO COMPARATIVO DE PROSTATECTOMÍA RADICAL ABIERTA RETROPUBICA, LAPAROSCÓPICA Y ROBÓTICA: RESULTADOS DE CONTINENCIA URINARIA Y FUNCIÓN SEXUAL (PARTE II).
}

\author{
Javier Romero Otero'y Juan I. Martínez-Salamanca².
}

'Department of Urology. Memorial Sloan-Kettering Cancer Center. New York. USA.

${ }^{2}$ Robotic Prostatectomy Program \& Urology Oncology Outcomes. Weill Medical College at Cornell University. New York Presbyterian Hospital. Brady Urological Institute. New York. USA.

\begin{abstract}
Resumen.- OBJETIVO: La prostatectomía radical es un procedimiento ampliamente aceptado y consolidado como tratamiento del cáncer de próstata clínicamente localizado. El resultado oncológico obtenido es excelente, por lo cual en la actualidad los resultados funcionales, en términos de continencia y función eréctil, se han convertido en factores determinantes a la hora de decidir el abordaje terapéutico. La prostatectomía radical abierta (PRA) es el "patrón oro" contra el que las nuevas técnicas: laparoscopia (PRL) y robótica (PRR) deben competir. Revisamos la literatura para comparar los tres abordajes en cuanto a resultados funcionales se refiere
\end{abstract}

MÉTODOS: Realizamos una búsqueda sistemática en: PubMed; EMBASE; Cochrane; SCOPUS; Science Citation Index durante el periodo 1990-Enero 2007 para

Javier Romero Otero

Alcalá, 233 3ㅁ

28028 Madrid. (España).

jromerootero@hotmail.com

Trabajo recibido: 26 de marzo 2007. los términos: "radical retropubic prostatectomy"; "open radical prostatectomy"; "laparoscopic prostatectomy"; "laparoscopic radical prostatectomy"; "robotic prostatectomy"; "robotic radical prostatectomy" and "functional assessment"; "continente"; "urinary function"; "incontinente"; "erectile function"; "sexual function"; "quality of life"; "functional assessment" y "minimally invasive treatment".

RESULTADOS: La comparación de resultados de distintas técnicas quirúrgicas se debería hacer mediante la realización de estudios randomizados. Ante la ausencia de éstos y la escasez de estudios comparativos no aleatorizados existentes, el único medio que nos queda es comparar las series más importantes para cada uno de los tres abordajes. Los resultados obtenidos por lo tanto son muy limitados. Aceptando la definición de continencia como la necesidad de O absorbentes/día y evaluado a los 12 meses, las tasas de continencia para cada uno de los abordajes: PRA, PRL y PRR son respectivamente: $81 \%$ (60-93\%); $87 \%(82-95 \%)$ y $91 \%$ (84-98\%). Para la función eréctil si consideramos como potente a aquel paciente capaz de completar el coito, con/sin ayuda de medicación oral y evaluado a los 12 meses; las tasas para PRA, PRL y PRR son respectivamente: 68\% (62-75\%), 69\% (52-78\%) y 60\% (20-97\%).

CONCLUSIONES: Para comparar los resultados de las distintas técnicas deberíamos desarrollar estudios prospectivos y aleatorios. Ante su ausencia al evaluar las series contemporáneas más significativas, destaca la ausencia de homogeneidad a la hora de medir y comunicar los resultados. Pese a estas limitaciones parece evidenciarse que no existen diferencias significativas entre los tres abordajes en cuanto a continencia y función eréctil se refiere. Es imperativo el desarrollo de estudios aleatorizados y la homogenización de criterios 
Palabras clave: Prostatectomía radical. Prostatectomía radical laparoscópica. Prostatectomía radical robótica. Incontinencia urinaria. Disfunción eréctil. Impotencia. Calidad de vida.

Summary.- OBJECTIVES: Radical prostatectomy is worldwide accepted as treatment for clinically localized prostate cancer. Its oncological results are excellent, so nowadays the functional outcomes: continence and potency, have become essential factors when evaluating the results. Open radical prostatectomy (ORP) is the gold standard against the new techniques, Laparoscopic (LRP) and robotic (RRP) must be compared. A systematic review of the literature is done to evaluate functional out comes between the three approaches.

METHODS: Systematic review in the databases: PubMed; EMBASE; Cochrane; SCOPUS; Science Citation Index for: "radical retropubic prostatectomy", "open radical prostatectomy"; "laparoscopic prostatectomy"; "laparoscopic radical prostatectomy"; "robotic prostatectomy"; "robotic radical prostatectomy and functional assessment"; "continence"; "urinary function"; "incontinence"; "erectile function"; 'sexual function"; "quality of life"; "functional assessment"; "minimally invasive treatment was performed".

RESULTS: The lack of randomized trials for this issue forces us to evaluate the functional results comparing the most important series of each approach, so the value of the results are very limited. Accepting 0 pads per day as continence definition and evaluated 12 months following surgery, the continence rates for each approach ORP, LRP and RRP are respectively: $81 \%$ (60-93\%); $87 \%(82-95 \%)$ and $91 \%$ (84-98\%). For erectile function admitting the capability for intercourse as potency definition, with or without the use of oral drugs and evaluated 12 months following surgery; the potency rates for each approach are: 68\% (62-75\%), 69\% (52-78\%) y 60\% (20-97\%).

CONCLUSIONS: Due the lack of randomized trials, we have to evaluate the most important contemporaneous series. The lack of homogeneity in evaluating and reporting results is evident in the urological community. With the limitations of the data available it seems to exist no differences between the three groups for functional out comes. It is mandatory to develop randomized trials and achieve a consensus for the criteria at the time of evaluating the functional outcomes.

Keywords: Radical prostatectomy. Laparoscopic radical prostatectomy. Robotic radical prostatectomy. Urinary incontinence. Erectile disfunction. Impotent. Quality of life.

\section{INTRODUCCIÓN}

La prostatectomía radical (PR) es un tratamiento ampliamente aceptado para el cáncer de próstata localizado. Desde la descripción de la técnica inicial se han desarrollado múltiples modificaciones (1). Una vez alcanzado y demostrado el control oncológico, el objetivo se ha fijado en los resultados funcionales. La PR no puede ser entendida solamente como una cirugía exerética, sino como una cirugía exerética y funcional (2). Tras la descripción de los haces neurovasculares por Walsh y Donker comenzó un nuevo camino en búsqueda de la preservación de la función eréctil y urinaria (3). Dado que hoy en día la mayoría de tumores que tratamos son de bajo riesgo, la mutilación funcional secundaria a su tratamiento parece un precio muy alto que pagar.

Para evaluar la importancia que los enfermos prostatectomizados conceden a los resultados funcionales, Hara y cols. (4) realizaron un estudio prospectivo. Utilizaron dos grupos con 52 y 54 enfermos intervenidos con prostatectomía radical laparoscópica (PRL) y prostatectomía radical abierta (PRA) respectivamente. A ambos les administraron tres cuestionarios para evaluar: 1) su estado de salud general (cuestionario de estado general de salud del European Organización for the Research and Treatment Group), 2) de salud sexual (cuestionario Internacional Index de la Función Eréctil 5) y 3) de función urinaria (cuestionario Internacional Continente Society Male SF), antes y después de ser intervenidos. Concluyen que mientras que no existen diferencias en el resultado del estado de salud general, sí que se aprecian en cuanto a la calidad de vida sexual y urinaria $(p<0.05)$, siendo éstos peores después de ambos procedimientos quirúrgicos. Estos resultados se refuerzan gracias al estudio de Steineck y cols. (5), quienes randomizaron 326 enfermos en dos grupos: observación y PR. La disfunción eréctil y la incontinencia fueron más frecuentes tras cirugía. Destaca la importancia que el enfermo otorga a los resultados funcionales el hecho de que ansiedad, depresión, bienestar general y la sensación personal de calidad de vida fueron iguales entre ambos grupos, es decir con y sin cirugía.

Ha pasado ya el suficiente tiempo desde que la PRL (6) y la robótica (7) (PRR) irrumpieran como opciones de abordaje en esta cirugía. Consecuentemente tenemos ya suficiente experiencia para poder evaluar cada uno de los abordajes en cuanto a resultados funcionales se refiere. El objetivo de este escrito es revisar todas las experiencias publicadas con el ánimo de poder extraer conclusiones en este campo. 


\section{MATERIAL Y MÉTODOS}

Realizamos una búsqueda sistemática en: PubMed; EMBASE; Cochrane; SCOPUS; Science Citation Index durante el periodo 1990- Enero 2007 para los términos: "radical retropubic prostatectomy"; "open radical prostatectomy"; "laparoscopic prostatectomy"; "laparoscopic radical prostatectomy"; "robotic prostatectomy"; "robotic radical prostatectomy"; "functional assessment"; "continence"; "urinary function"; "incontinence; erectile function"; "sexual function"; "quality of life"; "functional assessment" $y$ "minimally invasive treatment". El análisis esta restringido a artículos publicados en inglés. Analizamos las series más representativas para cada uno de los tres abordajes en cuanto a continencia y función eréctil se refiere.

\section{RESULTADOS}

El método ideal para comparar los tres abordajes quirúrgicos sería la realización de estudios prospectivos, aleatorizados, en una misma institución donde se utilizase la misma población, criterios clínicos y medidas de evaluación (8). En el momento actual no existe ningún tipo de estudio de estas características en la literatura. El hecho de que cada paciente y cirujano tengan sus preferencias a la hora de decidir el tipo de tratamiento hace que sea muy difícil el desarrollo de este tipo de estudios (8). Ante esta dificultad la siguiente opción es diseñar estudios prospectivos, no aleatorizados, dentro de una misma institución y comparar las 3 vías de abordaje, esto hasta donde llega nuestro conocimiento se ha realizado en un sólo centro (9) y es únicamente en relación a calidad de vida. Sí se han realizado estudios con este diseño pero comparando solamente dos de las técnicas quirúrgicas entre sí (10-16). En este escenario la mejor manera de poder valorar los resultados de las tres técnicas quirúrgicas es evaluando las series históricas más importantes para cada una de ellas.

\section{CONTINENCIA URINARIA}

Al comparar los resultados de las diferentes series de continencia urinaria tras PR nos encontramos ante multitud de limitaciones. En primer lugar todos los enfermos intervenidos con PRL ó PRR lo han sido a partir de 1997, con lo cual estos nuevos abordajes se han beneficiado de la experiencia quirúrgica acumulada a lo largo de los años, así deben compararse series contemporáneas (17).

En segundo lugar las poblaciones de las distintas publicaciones deberían ser homogéneas pues multitud de factores han sido relacionados con las tasas de continencia urinaria y la rapidez de recuperación de la misma. Así se han descrito 1)factores dependientes de las características generales de los enfermos: índice de masa corporal (18), volumen prostático $(19,20)$ y edad del paciente $(18,20,21)$. También existen 2) factores que dependen de variaciones anatómicas: características de los ligamentos pubo-perineales, musculatura periuretral (22) y de la inervación trigonal (23). Por último las 3) variaciones en la técnica de disección del espécimen también son determinantes en las tasas finales de continencia. Takenaka y cols. (24) comunicaron que la preservación de la fascia del elevador del ano protegía a este músculo y al rabdo-esfínter durante la disección del ápex prostático. Por su parte Sakai y cols. (25) encontraron que la preservación del cuello vesical era el único predictor de recuperación de continencia pasados uno y tres meses desde la cirugía. Dejar una mayor longitud del cabo uretral remanente, durante la PR, ha sido relacionado con una mayor y más pronta recuperación de la continencia (26). La preservación de los ligamentos pubo-prostáticos (27, 28), de las vesículas seminales (29) y de los haces neurovasculares (30) durante la cirugía también han sido documentados como factores que determinan la tasa de continencia final de estos enfermos.

Por último queda comentar que la falta de uniformidad en los métodos de medida (entrevista personal o telefónica, cuestionarios), en la escala de valoración de los enfermos (cuestionarios de muy diversos tipos, no unificados) y persona que juzga el nivel de continencia (paciente, médico, enfermera) entre las diferentes publicaciones aporta el último factor de confusión a la hora de poder valorar los resultados conjuntamente.

El método más aceptado para evaluar la continencia son cuestionarios de calidad de vida, completados por el enfermo y sin la influencia del cirujano $(31,32)$. La definición de continencia más extendida y objetiva es la necesidad de 0 absorbentes/día. El tiempo de evaluación debería ser de 12 meses, que es el tiempo aceptado por la mayoría de la comunidad para recuperar el control urinario, si bien hay autores que hablan de recuperación incluso 2 años tras la cirugía $(33,34)$.

Con esta definición la tasa de continencia para el grupo de PRA varía de 60.5-93\%(35-40) (Tabla I). La tasa media de continencia sería de $81.25 \%$ a los 12 meses. La continencia menor la presenta el estudio de Standford y cols. (37) que es el resultado de un estudio multi-institucional (Prostate Cancer Outcomes Study), lo que provoca que no se obtengan resultados tan buenos como pueden ser los de ins- 
tituciones de referencia (40) o de cirujanos con una gran experiencia $(35,38)$. Como muy bien reflejan Bianco y cols. la experiencia y el cirujano son factores fundamentales en los resultados de la cirugía (41).

La forma de evaluar y comunicar los resultados en las series de PRL es más uniforme, gracias a la experiencia acumulada en los trabajos previos de PRA.

La mayoría de los autores valoran sus resultados con un cuestionario y en un periodo de 12 meses, con la definición de 0 absorbentes al día. Siguiendo estos criterios las tasas de continencia varían de $82-95 \%$ (42-48). Mientras que la tasa media de continencia para el grupo de PRL siguiendo esta definición sería de $87.25 \%$. Cabe comentar que la tasa de continencia de $95 \%$ pertenece al grupo de Curto, Gaston y cols. (42), que utilizan sistemáticamente la técnica de disección intrafascial, lo que parece mejora los resultados funcionales, pero podría favorecer la presencia de márgenes positivos.

En el grupo de PRR en la mayoría de las series los resultados vienen referidos en un periodo de

TABLA I. RESULTADOS DE CONTINENCIA POST-PROSTATECTOMIAA DE LAS SERIES MÁS IMPORTANTES EXISTENTES EN LA LITERATURA PARA LAS TRES TÉCNICAS QUIRÚRGICAS.

\begin{tabular}{|c|c|c|c|c|c|c|}
\hline \multirow[t]{2}{*}{ Autor } & \multirow[t]{2}{*}{ Año } & \multirow{2}{*}{$\begin{array}{l}\text { № de } \\
\text { casos }\end{array}$} & \multirow{2}{*}{$\begin{array}{l}\text { Definición } \\
\text { Continencia }\end{array}$} & \multirow[t]{2}{*}{ Método de recogida } & \multicolumn{2}{|c|}{ Continencia } \\
\hline & & & & & Periodo & $\%$ \\
\hline \multicolumn{7}{|c|}{ Resultados series de PRA } \\
\hline Kundu (35) & 2004 & 2737 & 0 absorbentes & Cuestionario & $12 m$ & 93 \\
\hline Penson (36) & 2005 & 1213 & 0 absorbentes & Cuestionario & $12 m$ & 69 \\
\hline Potosky (34) & 2004 & 901 & 0 absorbentes & Cuestionario & $60 \mathrm{~m}$ & 71 \\
\hline Stanford (37) & 2000 & 1291 & 0 absorbentes & Cuestionario & $12 m$ & 60.5 \\
\hline Twiss (33) & 2005 & 373 & Pérdida casional & Cuestionario & $24 \mathrm{~m}$ & 96 \\
\hline Bianco (38) & 2005 & 1288 & 0 absorbentes & Entrevista & $12 \mathrm{~m}$ & 91 \\
\hline Harris (39) & 2006 & 92 & 0 absorbentes & Cuestionario & $12 \mathrm{~m}$ & 87 \\
\hline Saranchuck (40) & 2005 & 647 & 0 absorbentes & Cuestionario & $12 \mathrm{~m}$ & 87 \\
\hline \multicolumn{7}{|c|}{ Resultados series de PRL } \\
\hline Curto (42) & 2006 & 202 & 0 absorbentes & Cuestionario & $12 m$ & 95 \\
\hline Salomón (67) & 2002 & 100 & 0 absorbentes & Cuestionario & $12 m$ & 90 \\
\hline G. Alemán (48) & 2006 & 5824 & 0 absorbentes & Cuestionario & $12 m$ & 85 \\
\hline Rassweiler (45) & 2004 & 500 & 0 absorbentes & Cuestionario & $12 \mathrm{~m}$ & 83 \\
\hline Stolzenburg (46) & 2005 & 420 & 0 absorbentes & Cuestionario & $12 m$ & 92 \\
\hline Rozet (47) & 2005 & 599 & 0 absorbentes & Cuestionario & $12 m$ & 84 \\
\hline Turk (71) & 2001 & 125 & 1 absorbentes & Cuestionario & $9 m$ & 92 \\
\hline Guillonneau (44) & 2002 & 341 & 0 absorbentes & Cuestionario & $12 m$ & 82 \\
\hline Link (72) & 2005 & 122 & 0 absorbentes & Cuestionario & $12 m$ & 93 \\
\hline Brown (43) & 2005 & 75 & 1 absorbentes & Cuestionario & $12 \mathrm{~m}$ & 87 \\
\hline \multicolumn{7}{|c|}{ Resultados series de PRR } \\
\hline Menon (73) & 2003 & 200 & 1 absorbentes & Entrevista & $6 m$ & $96 \%$ \\
\hline Bentas (51) & 2003 & 41 & 1 absorbentes & Cuestionario & $12 m$ & $84 \%$ \\
\hline Ahlering (74) & 2003 & 45 & 1 absorbentes & Cuestionario & $3 m$ & $95 \%$ \\
\hline Patel (49) & 2005 & 200 & 0 absorbentes & Cuestionario & $12 m$ & $98 \%$ \\
\hline Joseph (54) & 2006 & 179 & 0 absorbentes & Entrevista & $6 m$ & $96 \%$ \\
\hline Kaul (75) & 2005 & 154 & 1 absorbente & Cuestionario & $12 m$ & $97 \%$ \\
\hline Menon (52) & 2006 & 2652 & 0 absorbentes & Cuestionario & $12 m$ & $84 \%$ \\
\hline Carlsson (76) & 2006 & 72 & 0 absorbentes & Cuestionario & $6 m$ & $90 \%$ \\
\hline
\end{tabular}


tiempo inferior a los 12 meses (Tabla I), como consecuencia de la reciente incorporación de esta técnica a la mayoría de los centros que la utilizan. Los autores pioneros en esta técnica sí tienen ya resultados con este periodo de seguimiento y comunican cifras que oscilan de $98-97 \%(49,50)$. La serie de Bentas y cols. (51) comunican hasta un $84 \%$ de pacientes continentes, pero utilizando el criterio de 0-1 absorbente al día. En la serie de robótica más amplia publicada hasta el día de hoy en la literatura Menon y cols. (52) comunican en 2652 pacientes unas tasas de continencia valoradas a los 12 meses de: $84 \%$ 0 absorbentes, $8 \% 1$ absorbente por seguridad pero no por necesidad, 3.2\% 1 absorbente por necesidad al día, 4\% 2-3 absorbentes al día y $0.8 \%$ totalmente incontinente. Como se puede apreciar son resultados muy similares a los esperables en las series equivalentes de PRA y de PRL. La mayoría de los autores con experiencia en robótica hacen un gran énfasis en que la recuperación de la función es muy rápida, así Menon (53) habla de recuperación del $95 \%$ de sus enfermos en 42 días y Joseph y cols. (54) del 93\% a los 3 meses.

Existen estudios en los que comparan los resultados de dos de estas técnicas entre sí (Tabla II). Comparando PRA y PRL Roumeguere y cols. (10) realizaron en la misma institución y de forma contemporánea 77 PRA vs $85 L R P$, la continencia alcanzada a los 12 meses, definida como el uso de 0 absorbentes al día era de $83.9 \%$ vs $80.7 \%$ ( $p>0.05$ ). Anastisiadis y cols. (12) realizaron un estudio con el mismo diseño y resultó $66.7 \%$ para las 70 PRA vs $71.6 \%$ en las 230 PRL $(p>0.05)$.

Comparando el abordaje abierto y robótico los dos estudios más significativos $(55,56)$ obtienen resultados muy similares. El estudio de Ahlering (55) es muy limitado dado que compara sus pacientes intervenidos mediante cirugía robótica con una serie de PRA histórica, además descarta las primeras PRR (lo justifica diciendo que es para eliminar la teórica curva de aprendizaje) y sólo hace referencia a los resultados pasados 3 meses de la cirugía. En el otro estudio Tewari y cols. (56) obtienen cifras de continencia muy similares pero se alcanzan antes en el grupo de la robótica $(p<0.05)$. Antiphon y cols. (57) comparando PRL vs PRR incluyó solamente 16 pacientes y no se apreciaron diferencias significativas.

\section{DISFUNCIÓN ERECTIL}

Poder hacer estudios sobre función eréctil es todavía si cabe más complejo. La función eréctil y más aún la satisfacción sexual tienen un gran componente subjetivo que dificulta encontrar un criterio de medida. Long, J.A. y cols. (58) administraron el cuestionario IIEF-5 (Index Internacional Erectil Function) sobre salud sexual a los enfermos que iban a ser sometidos a PR, encontraron que hasta un $65 \%$ de los enfermos cumplían los criterios de disfunción eréctil

TABLA II. RESULTADOS DE CONTINENCIA DE LOS ESTUDIOS COMPARATIVOS DE LAS DISTINTAS TÉCNICAS EXISTENTES EN LA LITERATURA.

\begin{tabular}{|c|c|c|c|c|c|c|}
\hline \multirow[t]{2}{*}{ Autor } & \multirow[t]{2}{*}{ Año } & \multirow{2}{*}{$\begin{array}{l}\text { № de } \\
\text { casos }\end{array}$} & \multirow{2}{*}{$\begin{array}{l}\text { Definición } \\
\text { Continencia }\end{array}$} & \multirow[t]{2}{*}{ Método de recogida } & \multicolumn{2}{|c|}{ Continencia } \\
\hline & & & & & Periodo & $\%$ \\
\hline \multicolumn{7}{|c|}{ Resultados series de PRA vs PRL } \\
\hline \multirow[t]{2}{*}{ Roumeguere(10) } & 2003 & 77 PRA & 0 absorbentes & & $12 m$ & 83.9 \\
\hline & 2003 & $85 \mathrm{PRL}$ & 0 absorbentes & & $12 m$ & 80.7 \\
\hline \multirow[t]{2}{*}{ Anastisiadis(12) } & 2003 & 70 PRA & 0 absorbentes & Cuestionario & $12 m$ & 66.7 \\
\hline & 2003 & 230PRL & 0 absorbentes & Cuestionario & $12 \mathrm{~m}$ & 71.6 \\
\hline \multicolumn{7}{|c|}{ Resultados series de PRA vs PRR } \\
\hline \multirow[t]{2}{*}{ Ahlering(55) } & 2004 & 60 PRA & 0 absorbentes & Cuestionario & $3 m$ & 76 \\
\hline & 2004 & 60 PRR & 0 absorbentes & Cuestionario & $3 m$ & 75 \\
\hline \multirow[t]{2}{*}{ Tewari(56) } & 2003 & 100PRA & 0 absorbentes & Cuestionario & $12 m$ & 92 \\
\hline & 2003 & 200PRR & 0 absorbentes & Cuestionario & $12 \mathrm{~m}$ & 93 \\
\hline \multicolumn{7}{|c|}{ Resultados series de PRR vs PRL } \\
\hline \multirow[t]{2}{*}{ Antiphon(57) } & 2003 & $16 \mathrm{PRL}$ & Pérdida mínima & Pérdida mínima & $12 m$ & 94 \\
\hline & 2003 & 16 PRR & Pérdida mínima & & $12 m$ & 81 \\
\hline
\end{tabular}


previo a cirugía. Además encontraron que la satisfacción sexual era dependiente de la rigidez axial del pene en los sujetos menores de 65 años, pero no así en los mayores de esta edad.

Para intentar definir y medir la función sexual de los sujetos se han intentado desarrollar incontables cuestionarios (IIEF, SHIM, UCLA...), reflejando la dificultad de la empresa. Sajonia y cols. (59) en 244 enfermos que afirmaban ser potentes en la entrevista médico-paciente, descubrió al administrar el cuestionario IEEF-5 que solamente el $45 \%$ de estos enfermos cumplían los criterios de potencia o ausencia de disfunción eréctil.

Por su parte Dubbelman y cols. (60) afirman que en los estudios en los que el instrumento de me- dida son entrevistas y cuestionarios no validados se obtienen las tasas de potencia más variables y más altas. Por otro lado no debemos olvidar que muchos de estos enfermos siguen tratamiento con inhibidores de la 5 fosofodiesterasa, inyecciones intracavernosas $u$ otros y agregan variabilidad a la hora de interpretar los resultados. Hoy en día está ampliamente aceptado el uso de medicación oral pero no de inyecciones. Además existen multitud de centros donde rutinariamente se realiza terapia rehabilitadora precoz de la función sexual mediante el uso de inyecciones intracavernosas.

Además hay una serie de factores que influyen de una manera objetiva y constante en la potencia tras prostatectomía. Muchas veces las poblaciones a estudio no pueden ser homogéneas en cuanto

TABLA III. RESULTADOS DE POTENCIA SEXUAL POST-PROSTATECTOMÍA DE LAS SERIES MÁS IMPORTANTES EXISTENTES EN LA LITERATURA PARA LAS TRES TÉCNICAS QUIRÚRGICAS.

\begin{tabular}{|c|c|c|c|c|c|}
\hline Autor & Año & $\begin{array}{l}\text { № de } \\
\text { casos }\end{array}$ & Definición Potencia & Recogida Datos & Potencia (\%) \\
\hline \multicolumn{6}{|c|}{ Resultados series de PRA } \\
\hline Kundu (35) & 2004 & 1834 & Coito & Cuestionario & $12 m=75 \%$ \\
\hline Penson (36) & 2005 & 1213 & Coito & Cuestionario & $12 m=17 \%$ \\
\hline Potosky (34) & 2004 & 901 & Coito & Cuestionario & $60 m=25 \%$ \\
\hline Twis (33) & 2005 & 200 & Coito & Cuestionario & $24 m=64 \%$ \\
\hline Bianco (38) & 2005 & 785 & Coito & Entrevista & $24 m=70 \%$ \\
\hline Saranchuck (40) & 2005 & 647 & Erección & Entrevista & $24=62 \%$ \\
\hline \multicolumn{6}{|c|}{ Resultados series de PRL } \\
\hline Curto (42) & 2006 & 137 & Coito & Cuestionario & $12 m=59 \%$ \\
\hline Salomón (77) & 2003 & 77 & Coito & Cuestionario & $12 m=59 \%$ \\
\hline German Grup (48) & 2006 & 5824 & Coito & Cuestionario & $12 m=52 \%$ \\
\hline Rassweiler (45) & 2004 & 41 & Coito & Cuestionario & $12 m=67 \%$ \\
\hline Stolzemburg (46) & 2005 & 83 & Coito & Cuestionario & $6 m=47 \%$ \\
\hline Rozet (47) & 2005 & 599 & Coito & Cuestionario & $6 m=43 \%$ \\
\hline Guillonneau (44) & 2002 & 41 & Coito & Cuestionario & $12 m=66$ \\
\hline Link (72) & 2005 & 122 & Coito & Cuestionario & $12 m=78 \%$ \\
\hline \multicolumn{6}{|c|}{ Resultados series de PRR } \\
\hline Menon (53) & 2003 & 100 & Coito & Cuestionario & $6 m=59 \%$ \\
\hline Bentas (51) & 2003 & 41 & Coito & Cuestionario & $12 m=20 \%$ \\
\hline Ahlering (78) & 2005 & 23 & Coito & Cuestionario & $3 m=47 \%$ \\
\hline Menon (69) & 2005 & 35 & Coito & Cuestionario & $12 m=97 \%$ \\
\hline Chien (70) & 2005 & 56 & Regreso a la situación basal & Cuestionario & $12 m=69 \%$ \\
\hline Joseph (54) & 2006 & 325 & IIEF > 21 & Cuestionario & $6 m=68 \%$ \\
\hline Kaul (50) & 2006 & 154 & Coito & Cuestionario & $12 m=96 \%$ \\
\hline \multirow[t]{2}{*}{ Menon (52) } & 2006 & 1113 & Coito & Cuestionario & $12 m=70 \%$ \\
\hline & & & & & $24 m=100 \%$ \\
\hline
\end{tabular}


a estos factores se refiere. Así hay una clara relación con: la edad, el estado de potencia sexual previo y la conservación de los haces neurovasculares durante la cirugía $(35,36,61-65)$. Existen otros factores más dudosos en la literatura: incontinencia urinaria, estenosis uretro-vesical, nivel de escolarización y estadio patológico $(62,63,65)$. Pero se ha llegado a relacionar incluso la edad de la pareja del sujeto con la recuperación sexual tras PR (66). Además no debemos olvidar el hecho de que la recuperación de la función eréctil es gradual y progresiva a lo largo del tiempo (62).

Por último quedaría comentar la influencia que tiene el diseño del estudio y el centro en el que se realiza. La mayoría de la literatura científica al valorar las tasas de función eréctil lo hace en base a estudios basados en poblaciones jóvenes, sexualmente activas y tratados por cirujanos o centros de gran experiencia (60). Pero cuando se analizan estudios de comunidades o centros no tan especializados encontramos resultados mucho menos espectaculares: Karakiewicz y cols. al valorar 2415 enfermos de Quebec encuentra solamente un $25 \%$ de enfermos potentes, Potosky (34) en 901 sujetos un $17.9 \%$ y Stanford (37) en 1291 individuos un 40.1\%. Dubbelman y cols. (60) concluyen en una revisión de la literatura que la potencia tras PR se podría definir como del $19 \%$.

En el análisis que vamos a desarrollar vamos a basarnos en las series más significativas de cada uno de los tres abordajes, puesto que no podemos comparar técnicas novedosas que se están desarrollando en centros muy especializados con la técnica de PRA que se efectúa en la mayoría de los centros existentes en todo el mundo. Debemos hacer referencia a la experiencia de centros y cirujanos del mismo nivel.

Así al evaluar los resultados obtenidos con la PRA en las series de los centros/cirujanos de mayor experiencia encontramos que varía de $62-75 \%$ (33, $35,38,40$ ) (Tabla III). Es interesante observar como Kundu y cols. (35) al valorar los resultados de la serie de PR realizadas entre 1983-2003 por Catalona, encuentra que para los primeros 1000 enfermos la recuperación de la función eréctil es del $68 \%$, mientras que en los sujetos del 1000 al 3000 es del $78 \%$, siendo estadísticamente significativa la diferencia $(p<0.0001)$.

Las series más importantes de PRL expresan de una forma bastante homogénea los resultados, definiendo potencia como la capacidad de mantener relaciones sexuales con/sin medicación oral, evaluada mediante un cuestionario y pasados 12 meses desde la cirugía. Así con este criterio la recuperación de la función sexual varía del 52-78\% $(42,44,45$, $48,67,68)$.

Siguiendo el mismo criterio de potencia, en el grupo de PRR la recuperación de la misma varía de $20-97 \%(51,52,69,70)$. Menon y cols. (69) compara su nueva técnica preservando la cara anterior de la fascia prostática, con la antigua técnica original del Instituto Vattikuti. La recuperación respetando la cara anterior de la fascia o "Velo de Afrodita" es del 97\%, mientras que con la técnica original es del $74 \%$.

Los estudios comparando PRA y PRL obtienen resultados muy similares. Roumeguere y cols. (10) con la definición de potencia de capacidad para mantener relaciones sexuales, recogido con un cuestionario y valorado a los 12 meses describe que en el grupo de PRA es del $54 \%$ vs $65 \%$ para el grupo de PRL. Anastisiadis y cols. (12) por su parte con la misma definición de potencia, pero sin establecer como recoge la información, comunica unos resultados para PRA vs PRL del $46-53 \%$ respectivamente a los 12 meses de la cirugía.

Tewari y cols. compararon 100 PRA vs 200 PRR en una sola institución y contemporáneamente. En los resultados que ofrece de potencia sexual no está incluida la proporción de sujetos que recuperan la función sexual al año de la cirugía. Sí refiere que el $50 \%$ de los sujetos intervenidos mediante PRR recuperaron la función eréctil a los 180 días, mientras que los que se operaron mediante PRA la recuperaron a los 440 días $(p<0.05)$.

\section{CONCLUSIONES}

Comparar los resultados de distintas técnicas quirúrgicas es difícil. Al querer valorar aspectos funcionales que repercuten en la calidad de vida de los enfermos, se añade un componente subjetivo, que aumenta la variabilidad de los resultados. En el momento actual es necesario establecer una definición, un modo de evaluación y un periodo de tiempo para continencia y potencia sexual tras prostatectomía radical. El modo ideal de valorar las distintas técnicas quirúrgicas sería desarrollar estudios aleatorizados y prospectivos. Ante la dificultad que esto presenta, solamente si todos recogemos la información y resultados de nuestros enfermos siguiendo los mismos criterios, podremos en un futuro comparar los resultados de los distintos grupos para obtener conclusiones válidas.

Con los datos existentes en la literatura no se pueden establecer diferencias con una base científica 
de sustento. Aceptando estas limitaciones, parece no haber una gran diferencia entre los tres abordajes en los resultados a largo plazo en cuanto a continencia urinaria y función eréctil se refiere.

\section{BIBLIOGRAFIA Y LECTURAS RECOMENDADAS ( ${ }^{*}$ lectura de interés $y^{* *}$ lectura fundamental)}

1. YOUNG, H.: "The early diagnosis and radical cure of carcinoma of the prostate". Bull Hopkins University. 175: 315, 1905.

2. WALSH, P.C.: "Anatomic radical prostatectomy: evolution of the surgical technique". J. Urol., 160: 2418, 1998.

*3. WALSH, P. C. y DONKER, P. J.: "Impotence following radical prostatectomy: insight into etiology and prevention”. J. Urol. 128: 492, 1982.

4. HARA, I. y cols.: "Comparison of quality of life following laparoscopic and open prostatectomy for prostate cancer". J. Urol. 169:2045, 2003.

5. STEINECK, G. y cols.: "Quality of life after radical prostatectomy or watchful waiting". N. Engl. J. Med.. 347: 790, 2002.

**6. GUILLONNEAU, B. y VALLANCIEN, G.: "Laparoscopic radical prostatectomy: the Montsouris technique". J. Urol. 163: 1643, 2000.

7. ABBOU, C.C. y cols.: "Laparoscopic radical prostatectomy with a remote controlled robot. J. Urol. 165: 1964, 2001.

8. LEPOR, H. "Open versus Laparoscopic Radical Prostatectomy". Rev. Urol. 7: 115, 2005.

9. BALL, A. J. y cols.: "Prospective longitudinal comparative study of early health-related qualityof-life outcomes in patients undergoing surgical treatment for localized prostate cancer: a shortterm evaluation of five approaches from a single institution". J. Endourol. 20: 723, 2006.

*10. ROUMEGUERE, T. y cols.: "Radical prostatectomy: a prospective comparison of oncological and functional results between open and laparoscopic approaches". World J. Urol. 20: 360, 2003.

11. RASSWEILER, J. y cols.: "Laparoscopic versus open radical prostatectomy: a comparative study at a single institution. J. Urol. 169: 1689, 2003.

*12. ANASTASIADIS, A. G. y cols.: "Radical retropubic versus laparoscopic prostatectomy: a prospective comparison of functional outcome. Urology. 62: 292, 2003.

13. MENON, M. y cols.: "Prospective comparison of radical retropubic prostatectomy and robot-assisted anatomic prostatectomy: the Vattikuti Urology Institute experience". Urology. 60: 864, 2002.

14. MENON, M. A.; SHRIVASTAVA, y TEWARI, A.: "Laparoscopic radical prostatectomy: conventional and robotic". Urology. 66: 101, 2005.
15. WEBSTER, T.M. y cols.: "Robotic assisted laparoscopic radical prostatectomy versus retropubic radical prostatectomy: a prospective assessment of postoperative pain". J. Urol. 174:912, discussion 914., 2005.

16. JOSEPH, J.V. y cols.: "Robot-assisted vs pure laparoscopic radical prostatectomy: are there any differences?”. BJU Int. 96: 39, 2005.

17. SALOMON, L. y cols.: "Open versus laparoscopic radical prostatectomy: part I". BJU Int. 94: 238, 2004.

18. EASTHAM, J. A. y cols.: "Risk factors for urinary incontinence after radical prostatectomy". J. Urol. 156:1707, 1996.

19. HSU, E.I.; HONG, E.K. y LEPOR, H.: "Influence of body weight and prostate volume on intraoperative, perioperative, and postoperative outcomes after radical retropubic prostatectomy". Urology 61: 601, 2003.

20. RODRIGUEZ, E.; SKARECKY, D.W. y AHLERING, T.E.: "Post-robotic prostatectomy urinary continence: characterization of perfect continence versus occasional dribbling in pad-free men". Urology 67: 785, 2006.

21. LICHT, M.R. y cols.: "Impact of bladder neck preservation during radical prostatectomy on continence and cancer control". Urology 44: 883, 1994.

22. MYERS, R. P. y cols.: "Puboperineales: muscular boundaries of the male urogenital hiatus in 3D from magnetic resonance imaging". J. Urol. 164: 1412,2000

23. JOHN, H. y cols.: "Evidence of trigonal denervation and reinnervation after radical retropubic prostatectomy. J Urol. 165: 111, 2001.

24. TAKENAKA, A. y cols.: "Preservation of the Puboprostatic Collar and Puboperineoplasty for Early Recovery of Urinary Continence after Robotic Prostatectomy: Anatomic Basis and Preliminary Outcomes. Eur Urol, 2006.

25. SAKAI, I. y cols.: "Intussusception of the bladder neck does not promote early restoration to urinary continence after non-nerve-sparing radical retropubic prostatectomy. Int J Urol. 12: 275, 2005.26.

26. VAN RANDENBORGH, H. y cols.: "Improved urinary continence after radical retropubic prostatectomy with preparation of a long, partially intraprostatic portion of the membraneous urethra: an analysis of 1013 consecutive cases". Prostate Cancer Prostatic Dis. 7: 253, 2004.

27. STEINER, M.S.; MORTON, R.A. y WALSH, P.C.: "Impact of anatomical radical prostatectomy on urinary continence. J. Urol. 145: 512; discussion 514, 1991.

28. STEINER, M.S.: "The puboprostatic ligament and the male urethral suspensory mechanism: an anatomic study”. Urology, 44: 530, 1994. 
29. HOLLABAUGH, R.S. Jr. y cols.: "Preservation of putative continence nerves during radical retropubic prostatectomy leads to more rapid return of urinary continence". Urology, 51: 960, 1998.

30. O'DONNELL, P. D. y FINAN, B.F.: "Continence following nerve-sparing radical prostatectomy". J. Urol. 142: 1227; discussion 1229, 1989.

31. JT, W. y M. JE, "Comparision of patients'and physicians'rating of urinary incontinence following radical prostatectomy". Semin Urol Oncol 18: 76, 2000.

32. MS, L., H. RD, AND F. A, "Quality of life outcomes in men treated for localized prostate cancer". JAMA 273:129, 1995.

33. TWISS, C.; SLOVA, D. y LEPOR, H.: "Outcomes for men younger than 50 years undergoing radical prostatectomy". Urology 66:141, 2005.

34. POTOSKY, A.L. y cols.: "Five-year outcomes after prostatectomy or radiotherapy for prostate cancer: the prostate cancer outcomes study". J. Natl. Cancer Inst. 96: 1358, 2004.

**35. KUNDU, S.D. y cols.: "Potency, continence and complications in 3,477 consecutive radical retropubic prostatectomies". J. Urol. 172: 2227, 2004.

**36. PENSON, D.F. y cols.: "5-year urinary and sexual outcomes after radical prostatectomy: results from the prostate cancer outcomes study". J. Urol. 173:170, 2005.

37. STANFORD, J. L. y cols.: "Urinary and sexual function after radical prostatectomy for clinically localized prostate cancer: the Prostate Cancer Outcomes Study". Jama 283:354, 2000.

**38. BIANCO, F.J. Jr.; SCARDINO, P.T. y EASTHAM, J.A.: "Radical prostatectomy: long-term cancer control and recovery of sexual and urinary function ("trifecta")". Urology, 66: 832005.

39. HARRIS, M.J., The Anatomic Radical Perineal Prostatectomy: An Outcomes-Based Evolution. Eur Urol, 2006.

40. SARANCHUK, J.W. y cols.: "Achieving optimal outcomes after radical prostatectomy". J. Clin. Oncol. 23: 4146, 2005.

41. BIANCO, F.J., Jr. y cols.: "Variations among high volume surgeons in the rate of complications after radical prostatectomy: further evidence that technique matters". J. Urol. 173: 2099, 2005.

**42. CURTO, F. y cols.: "Nerve sparing laparoscopic radical prostatectomy: our technique". Eur. Urol. 49:344, 2006.

43. BROWN, J.A. y cols.: "Transperitoneal versus extraperitoneal approach to laparoscopic radical prostatectomy: an assessment of 156 cases". Urology 65:320, 2005.

**44. GUILLONNEAU, B. y cols.: "Laparoscopic radical prostatectomy: assessment after 550 procedures". Crit Rev Oncol Hematol. 43:123, 2002.

45. RASSWEILER, J. y cols.: "Laparoscopic radical prostatectomy: functional and oncological outcomes". Curr. Opin. Urol. 14:75, 2004.

46. STOLZENBURG, J.U. y cols.: "Endoscopic extraperitoneal radical prostatectomy: oncological and functional results after 700 procedures". J. Urol. 174:1271; discussion 1275., 2005.

47. ROZET, F. y cols.: "Extraperitoneal laparoscopic radical prostatectomy: a prospective evaluation of 600 cases". J. Urol. 174:908, 2005.

**48. RASSWEILER, J. y cols.: "Laparoscopic radical prostatectomy--the experience of the German Laparoscopic Working Group. Eur Urol. 49: 113, 2006.

**49. PATEL, V. R. y cols.: "Robotic radical prostatectomy in the community setting--the learning curve and beyond: initial 200 cases". J. Urol. 174: 269, 2005.

50. KAUL, S. y cols.: "Functional outcomes and oncological efficacy of Vattikuti Institute prostatectomy with Veil of Aphrodite nerve-sparing: an analysis of 154 consecutive patients". BJU Int. 97:467, 2006.

51. BENTAS, W. y cols.: "Robotic technology and the translation of open radical prostatectomy to laparoscopy: the early Frankfurt experience with robotic radical prostatectomy and one year follow-up". Eur Urol. 44:175, 2003.

**52. MENON, M. y cols.: "Vattikuti Institute Prostatectomy: Contemporary Technique and Analysis of Results". Eur. Urol., 2006.

53. MENON, M. y cols.: "Vattikuti Institute Prostatectomy: a single-team experience of 100 cases. J Endourol. 17:785, 2003.

**54. JOSEPH, J.V. y cols.: "Robotic extraperitoneal radical prostatectomy: an alternative approach". J Urol. 175:945; discussion 951., 2006.

*55. AHLERING, T.E. y cols.: "Robot-assisted versus open radical prostatectomy: a comparison of one surgeon's outcomes". Urology, 2004. 63(5): p. 819-22.

*56. TEWARI, A.; SRIVASATAVA, A. y MENON, M.: "A prospective comparison of radical retropubic and robot-assisted prostatectomy: experience in one institution". BJU Int. 92:205, 2003.

57. ANTIPHON, P. y cols.: "Complete solo laparoscopic radical prostatectomy: initial experience". Urology. 61:724; discussion 728, 2003.

58. LONG, J.A. y cols.: "Evaluation of sexuality and erectile function of candidates for radical prostatectomy". Prog. Urol. 16:450, 2006.

59. SALONIA, A. y cols.: "Baseline potency in candidates for bilateral nerve-sparing radical retropubic prostatectomy". Eur. Urol. 50:360, 2006.

60. DUBBELMAN, Y.D.; DOHLE, G.R. y SCHRODER, F.H.: "Sexual function before and after radical retropubic prostatectomy: A systematic review of prognostic indicators for a successful outco- 
me". Eur. Urol. 50: 711; discussion 718, 2006.

61. RABBANI, F. y cols.: "Factors predicting recovery of erections after radical prostatectomy". J. Urol 164:1929, 2000.

62. QUINLAN, D.M. y cols.: "Sexual function following radical prostatectomy: influence of preservation of neurovascular bundles. J. Urol. 145: 998, 1991.

63. CATALONA, W. J. y BASLER, J.W.: "Return of erections and urinary continence following nerve sparing radical retropubic prostatectomy". J. Urol. 150:905, 1993.

64. CATALONA, W.J. y cols.: "Potency, continence and complication rates in 1,870 consecutive radical retropubic prostatectomies". J. Urol. 162: 433, 1999.

65. GEARY, E.S. y cols.: "Nerve sparing radical prostatectomy: a different view". J. Urol. 154:145, 1995.

66. DESCAZEAUD, A.; DEBRE, B. y FLAM, T. A.: "Age difference between patient and partner is a predictive factor of potency rate following radical prostatectomy". J. Urol. 176:2594; discussion 2598., 2006.

67. SALOMON, L. y cols.: "Urinary continence and erectile function: a prospective evaluation of functional results after radical laparoscopic prostatectomy". Eur. Urol. 42:338, 2002.

68. LINK, R.E. y cols.: "Making ends meet: a cost comparison of laparoscopic and open radical retropubic prostatectomy". J. Urol. 172:269, 2004.

69. MENON, M. y cols.: "Potency following robotic radical prostatectomy: a questionnaire based analysis of outcomes after conventional nerve sparing and prostatic fascia sparing techniques". J. Urol. 174:2291, discussion 2296., 2005.
70. CHIEN, G.W. y cols.: "Modified clipless antegrade nerve preservation in robotic-assisted laparoscopic radical prostatectomy with validated sexual function evaluation". Urology 66:419, 2005.

71. TURK, I. y cols.: "Laparoscopic radical prostatectomy. Technical aspects and experience with 125 cases". Eur Urol. 40:46; discussion 53., 2001.

72. LINK, R.E. y cols.: "Health related quality of life before and after laparoscopic radical prostatectomy". J. Urol. 173:175; discussion 179., 2005.

73. MENON, M. y TEWARI, A.: "Robotic radical prostatectomy and the Vattikuti Urology Institute technique: an interim analysis of results and technical points". Urology 61:15, 2003.

74. AHLERING, T. E. y cols.: "Successful transfer of open surgical skills to a laparoscopic environment using a robotic interface: initial experience with laparoscopic radical prostatectomy”. J. Urol. 170:1738, 2003.

75. KAUL, S.; SHAH, N.L. y MENON, M.: "Learning curve using robotic surgery. Curr Urol Rep, $7: 125,2006$.

76. CARLSSON, S.; NILSSON, A. y WIKLUND, P. N.: "Postoperative urinary continence after robot-assisted laparoscopic radical prostatectomy". Scand. J. Urol. Nephrol. 40:103, 2006.

77. SALOMON, L., y cols.: "Combined reporting of cancer control and functional results of radical prostatectomy". Eur. Urol. 44:656, 2003.

78. AHLERING, T.E.; EICHEL, L. y SKARECKY, D.: "Rapid communication: early potency outcomes with cautery-free neurovascular bundle preservation with robotic laparoscopic radical prostatectomy”. J. Endourol. 19:715, 2005. 